\title{
AutoWork 2019: Workshop on the Future of Work and Well-Being in Automated Vehicles
}

Permission to make digital or hard copies of part or all of this work for personal or

classroom use is granted without fee provided that copies are not made or distributed for profit or commercial advantage and that copies bear this notice and the full citation on the first page. Copyrights for third-party components of this work must be honored. For all other uses, contact the Owner/Author.

AutomotiveUl '19 Adjunct, September 21-25, 2019, Utrecht, Netherlands

(02019 Copyright is held by the owner/author(s).

ACM ISBN 978-1-4503-6920-6/19/09

http://dx.doi.org/10.1145/3349263.3350759
Andrew L. Kun

University of New Hampshire

Durham, NH 03824, USA

andrew.kun@unh.edu

\section{Orit Shaer}

Wellesley College

Wellesley, MA 02481, USA

oshaer@wellesley.edu

Stephen Brewster

University of Glasgow

Glasgow, G12 8QQ, UK

stephen.brewster@glasgow.ac.uk

Clemens Schartmüller

Technische Hochschule

Ingolstadt (THI)

85049 Ingolstadt, Germany

Johannes Kepler University

4020 Linz, Austria

clemens.schartmueller@thi.de

eas Riener

nische Hochschule

85049 Ingolstadt, Germany

andreas.riener@thi.de

\begin{abstract}
Automated vehicles will allow users to engage in non-driving activities related to work and well-being. This workshop will explore a number of questions related to human-computer interaction in vehicles with the ultimate goal of allowing users to be productive in automated vehicles, as well as to engage in activities that successfully increase their wellbeing. Additionally, the organizers will pilot a novel format for hybrid engagement of participants, which will include online activities before and after the workshop, as well as in-person activities at the workshop.
\end{abstract}

\section{Author Keywords}

Automated Driving; Human-Computer Interaction; Work; Well-being; Safety

\section{CCS Concepts}

-Human-centered computing $\rightarrow$ Human computer interaction $(\mathrm{HCl})$; $\cdot$ Applied computing $\rightarrow$ Computers in other domains;

\section{Introduction}

The US Census Bureau reported that in the US workers spend an average of about 50 minutes a day commuting to and from work [8]. In many of the world's major cities, commute time is over an hour. The impact on individuals, organizations, and society at large is enormous: millions 


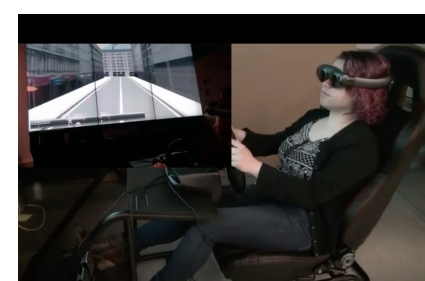

Figure 1: Investigation of augmented reality-supported activities in a simulated automated vehicle. of workers spend about 4 hours per week in vehicles with an economic cost in the US alone of $\$ 90$ billion per year [18]. Research also indicates that people with long commutes are more exhausted, less productive at work, and have lower job satisfaction [3].

With the advent of automation, users will increasingly engage in work and play in vehicles [9], and for this reason, we can start to address the challenges associated with commuting. Time spent driving the car will be transformed to productive working hours (and thereby in-office hours could get reduced) or to relaxation time, ultimately potentially improving work-life balance. Additionally, professions endangered by automated vehicles, like truck drivers, could be retained by having e.g., logistics-planning performed during the actual drive. However, for an automated vehicle to become a place of productivity, we need to understand how technology can allow to safely combine activities related to work and well-being with those related to driving. This is of utmost importance considering that specifically the very next generation of vehicles, i.e., conditionally automated vehicles as specified in SAE level 3 (cf. SAE J3016, [20]), require the human to be able to resume manual driving upon a take-over request by the automation system. At the same time, they promise to free the driver-passenger from the burden of observing the driving situation at all times, and thereby implicitly suggest that he or she is be able to engage in other tasks.

Accordingly, recent publications at prominent venues like $\mathrm{CHI}[6,13]$ or MobileHCl $[15,16]$ show the increasing research interest in the topic. At AutomotiveUI 2018, a Workshop on The Mobile Office was held [1] and resulted in an exploration of requirements, on which this workshop will build. Amongst many more, safety, ergonomics, comfort, and awareness, but also work-life effects and emotional as- pects have to be considered in the quest for an actually beneficial mobile office automated vehicle [21]. We will therefore further explore the human-computer interaction issues related to this emerging way that we will use vehicles and specifically focus on means to make the vision a reality.

\section{Workshop Goals}

The overarching goal of this workshop is to provide an avenue for researchers exploring the future of work in automated vehicles to exchange ideas, and to start charting a path towards increasing productivity and well-being for the users of automated vehicles, while at the same time maintaining safety. In particular, we will examine the use of technologies such as augmented reality (cf., Figure 1), in-vehicle speech interaction, and tangible interfaces (cf., Figure 2). More specifically, we will address the following issues:

1. Non-driving related tasks. Which tasks are of value for the productivity and/or well-being of users of automated vehicles? Which tasks are appropriate for the relatively-small cockpit of the car, and which ones can be safely performed so that the user can return to driving if needed? Which tasks are appropriate for the context in which the user's attention needs to switch relatively often between the non-driving task and driving?

2. Technologies. Which technologies can support the users as they work and play in vehicles? How can these technologies support the transition of control from the automation to the driver, and back again from the driver to the automation? Furthermore, how do we minimize the occurrence of motion sickness?

3. Ergonomics. What are interior and interface design requirements to ensure comfort and usability, as well 


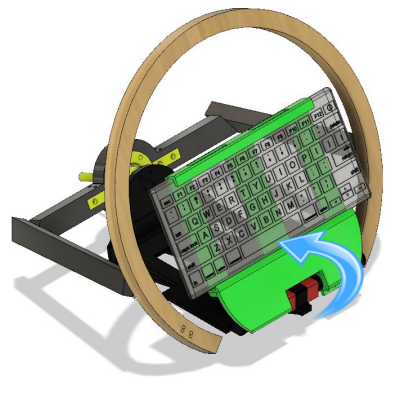

Figure 2: Steering wheel prototype that supports both take-overs and productive typing in highly automated driving [17].

\section{as productivity and safety?}

4. Evaluation techniques. What are the instruments and measures of evaluation? One key question is how to generalize results from the lab to expected results in real, on-road situations? Or, how can we test effectively in higher fidelity environments?

5. Road-map for productivity and well-being across different levels of automation. Which collaborations and funding mechanisms will help our community move forward effectively and efficiently in creating invehicle interfaces to support work and well-being in automated vehicles?

\section{Hybrid Engagement}

We intend to combine pre- and post-workshop activities with those during the actual half-day workshop, in order to guarantee valuable outcomes for the field of research.

1. Reflection statements and online presentations before the workshop. We will invite (voluntary) reflection statements (2-4 page EA format) on the topics above, from researchers and practitioners. The organizers will review the reflection statements and make decisions about acceptance. Authors of accepted reflection statements will be asked to submit a 3-5 minute video presentation. We will share all videos via Slack (or similar). Participants will be asked to view all presentations, comment on at least three of them, and respond to all comments on their own presentation.

2. Interactive activities at the workshop. At the workshop we will begin with team-building exercises. We will continue with small-, and large-group discussions, co-design, and prototyping, and collaborative writing.
3. Online reflection presentation after the workshop. We will ask participants to submit a 3-5 minute video summarizing their own takeaways from their unique perspectives. We will again share the videos and encourage participants to engage each other through comments. We plan to keep this online communication channel open for the community until the next AutomotiveUl conference, when we will assess progress and make further plans.

4. Technical report after the workshop. The organizers will report on the outcomes through blog posts on the workshop website.

5. Case study on hybrid engagement. The organizers will report on the hybrid engagement aspect of the workshop through a case-study submission to $\mathrm{CH}$ 2020. This submission will be made in light of recent discussions in the SIGCHI conferences board (Kun is a member), regarding possible future approaches to conducting SIGCHI specialized conferences. Thus, our experiences with hybrid engagement will be of value to the broader SIGCHI community.

\section{Workshop Organization}

The organizers will pro-actively perform recruiting and dissemination activities to provide a highly impacting workshop.

1. Recruitment. The five organizers will actively recruit participants to submit reflection statements. We will target both established experts and emerging scholars in our field. We will reach out to our contacts in academia, industry, and government.

2. Publicity. All material related to this workshop will be made available through the dedicated workshop 
website: http://cs.wellesley.edu/ mobileoffice/autoui. It contains the information relevant for people interested in the workshop, as well as submission information for authors of reflection statements. It will further include background material and related work, organizers' information, as well as a detailed schedule of the workshop. Accepted reflection statements will be made available for download (subject to approval by authors). The call for participation will be distributed through the $\mathrm{CHI} / \mathrm{UBICOMP}$ mailing lists, the Driving Assessment (DA) mailing list, the AutomotiveUI social media channels, and others.

\section{Organizers}

Andrew L. Kun is associate professor of Electrical and Computer Engineering at the University of New Hampshire. His research focus is human-computer interaction in vehicles $[4,5]$, primarily in speech interaction, as well as the use of visual behavior and pupil diameter measures to assess and improve the design of user interfaces. He served as the General Chair of the 2012 AutomotiveUI conference.

Orit Shaer is an Associate Professor of computer science and director of Media Arts and Sciences at Wellesley College. Her expertise is in designing, implementing and evaluating novel human-computer interactions including multitouch, augmented reality [12, 23], and tangible interaction $[19,22]$. In 2012 she was general chair of the ACM Interactive Surfaces and Spaces conference, in 2017 she served as program co-chair of the ACM Tangible Embedded and Embodied Interaction (TEI) conference, and in 2020 she will again serve as program co-chair of TEI.

Andreas Riener is a professor for Human-Machine Interaction and Virtual Reality at Technische Hochschule Ingolstadt (THI), Germany with co-appointment at CARISSMA
(Center of Automotive Research on Integrated Safety Systems and Measurement Area). He is further leading the human-computer interaction group ( $\mathrm{HClG}$ ) at THI. His research interests include driving ergonomics, driver state estimation from physiological measures, human factors in driver-vehicle interfaces (e.g., [14]), application of AR [24], as well as topics related to (over)trust and acceptance [25], and ethical issues [2] in automated driving.

Stephen Brewster is a professor of Human-Computer Interaction at the University of Glasgow, where he leads the Multimodal Interaction Group. His research focuses on using the range of human sensing and control capabilities to enable rich interactions between humans and technology. His automotive work focuses on designing cues to support handover [11], haptic and multimodal interaction in the car [10] and mitigating motion sickness when using VR in vehicles [7]. He is a member of the SIGCHI Academy and ACM Distinguished Speaker.

Clemens Schartmüller is a research assistant and $\mathrm{PhD}$ candidate in the research group of Prof. Riener at THI, in cooperation with the Johannes Kepler University Linz. In his $\mathrm{PhD}$, he conducts prototype-driven research on novel user interfaces for productive and safe office work in highly automated vehicles [16, 17].

\section{Acknowledgements}

Andrew Kun and Orit Shaer were supported in part by NSF grant CMMI-1840085. Andreas Riener and Clemens Schartmüller were supported by the "Innovative Hochschule" program of the German Federal Ministry of Education and Research (BMBF) under Grant No. 03IHS109A (MenschINBewegung). 


\section{REFERENCES}

1. Lewis L. Chuang, Stella F. Donker, Andrew L. Kun, and Christian P. Janssen. 2018. Workshop on The Mobile Office. In Adjunct Proceedings of the 10th International Conference on Automotive User Interfaces and Interactive Vehicular Applications (AutomotiveUl '18). ACM, New York, NY, USA, 10-16. DOI : http://dx.doi.org/10.1145/3239092.3239094

2. Anna-Katharina Frison, Philipp Wintersberger, and Andreas Riener. 2016. First Person Trolley Problem: Evaluation of Drivers' Ethical Decisions in a Driving Simulator. In Adjunct Proceedings of the 8th International Conference on Automotive User Interfaces and Interactive Vehicular Applications (AutomotiveUI '16 Adjunct). ACM, New York, NY, USA, 117-122. DOI :

http://dx.doi.org/10.1145/3004323.3004336

3. Francesca Gino, Bradley Staats, Jon M. Jachimowicz, Julia Lee, and Jochen I. Menges. 2017. Reclaim Your Commute. Harvard Business Review (2017), 149-153. https://hbr.org/2017/05/reclaim-your-commute Date accessed: 2019-08-09.

4. Andrew L. Kun. 2018. Human-Machine Interaction for Vehicles: Review and Outlook. Foundations and Trends ${ }^{\circledR}$ in Human-Computer Interaction 11, 4 (2018), 201-293. DOI :

http://dx.doi.org/10.1561/1100000069

5. A. L. Kun, S. Boll, and A. Schmidt. 2016. Shifting Gears: User Interfaces in the Age of Autonomous Driving. IEEE Pervasive Computing 15, 1 (Jan 2016), 32-38. DOI :

http://dx.doi.org/10.1109/MPRV.2016.14
6. Nikolas Martelaro, Jaime Teevan, and Shamsi T. Iqbal. 2019. An Exploration of Speech-Based Productivity Support in the Car. In Proceedings of the $2019 \mathrm{CHI}$ Conference on Human Factors in Computing Systems (CHI '19). ACM, New York, NY, USA, Article 264, 12 pages. DOI :

http://dx.doi.org/10.1145/3290605.3300494

7. Mark McGill, Alexander Ng, and Stephen Brewster. 2017. I Am The Passenger: How Visual Motion Cues Can Influence Sickness For In-Car VR. In Proceedings of the $2017 \mathrm{CHI}$ Conference on Human Factors in Computing Systems (CHI '17). ACM, New York, NY, USA, 5655-5668. DOI :

http://dx.doi.org/10.1145/3025453.3026046

8. Brian McKenzie and Melanie Rapino. 2011.

Commuting in the united states: 2009. US Department of Commerce, Economics and Statistics Administration, US.

9. Bastian Pfleging, Maurice Rang, and Nora Broy. 2016. Investigating User Needs for Non-Driving-Related Activities During Automated Driving. In Proceedings of the 15th International Conference on Mobile and Ubiquitous Multimedia. 91-99. DOI :

http://dx.doi.org/10.1145/3012709.3012735

10. Ioannis Politis, Stephen Brewster, and Frank Pollick. 2013. Evaluating Multimodal Driver Displays of Varying Urgency. In Proceedings of the 5th International Conference on Automotive User Interfaces and Interactive Vehicular Applications (AutomotiveUl '13). ACM, New York, NY, USA, 92-99. DOI :

http://dx.doi.org/10.1145/2516540.2516543 
11. Ioannis Politis, Stephen Brewster, and Frank Pollick. 2015. Language-based Multimodal Displays for the Handover of Control in Autonomous Cars. In Proceedings of the 7th International Conference on Automotive User Interfaces and Interactive Vehicular Applications (AutomotiveUI '15). ACM, New York, NY, USA, 3-10. DOI :

http://dx.doi.org/10.1145/2799250.2799262

12. Christina Pollalis, Elizabeth Joanna Minor, Lauren Westendorf, Whitney Fahnbulleh, Isabella Virgilio, Andrew L. Kun, and Orit Shaer. 2018. Evaluating Learning with Tangible and Virtual Representations of Archaeological Artifacts. In Proceedings of the Twelfth International Conference on Tangible, Embedded, and Embodied Interaction (TEl '18). ACM, New York, NY, USA, 626-637. DOI :

http://dx.doi.org/10.1145/3173225.3173260

13. Kathrin Pollmann, Oilver Stefani, Amelie Bengsch, Matthias Peissner, and Mathias Vukelić. 2019. How to Work in the Car of the Future?. In Proceedings of the 2019 CHI Conference on Human Factors in Computing Systems - CHI '19. 1-14. DOI : http://dx.doi.org/10.1145/3290605.3300284

14. Andreas Riener, Myounghoon Jeon, Ignacio Alvarez, and Anna K. Frison. 2017. Driver in the Loop: Best Practices in Automotive Sensing and Feedback Mechanisms. Springer International Publishing, Cham, 295-323. DOI :

http://dx.doi.org/10.1007/978-3-319-49448-7_11

15. Clemens Schartmüller and Andreas Riener. 2018. Safe, Productive and Socially Accepted Text Input in Highly Automated Driving. In MobileHCl 2018
Workshop on Socio-Technical Aspects of Text Entry. ceur-ws, Barcelona, Spain, 18-23.

http://ceur-ws.org/Vol-2183/position3.pdf

16. Clemens Schartmüller, Andreas Riener, Philipp Wintersberger, and Anna-Katharina Frison. 2018. Workaholistic: On Balancing Typing- and Handover-performance in Automated Driving. In Proceedings of the 20th International Conference on Human-Computer Interaction with Mobile Devices and Services (MobileHCl '18). ACM, New York, NY, USA, Article 16, 12 pages. DOI :

http://dx.doi.org/10.1145/3229434.3229459

17. Clemens Schartmüller, Philipp Wintersberger, Anna-Katharina Frison, and Andreas Riener. 2019. Type-o-Steer: Reimagining the Steering Wheel for Productive Non-Driving Related Tasks in Conditionally Automated Vehicles. In Proceedings of the 30th IEEE Intelligent Vehicles Symposium, IV'19. IEEE Intelligent Transportation Systems Society, Paris, France, In Press.

18. David Schrank, Bill Eisele, Tim Lomax, and Jim Bak 2015. 2015 URBAN MOBILITY SCORECARD. Technical Report. Texas A\&M Transportation Institute. https://d2dt15nnlpfrOr.cloudfront.net/tti.tamu. edu/documents/mobility-scorecard-2015.pdf Date accessed: 2019-08-09.

19. Orit Shaer, Eva Hornecker, and others. 2010. Tangible user interfaces: past, present, and future directions. Foundations and Trends ${ }^{\circledR}$ in Human-Computer Interaction 3, 1-2 (2010), 4-137. DOI : http://dx.doi.org/10.1561/1100000026 
20. SAE Society of Automotive Engineers. 2018. Taxonomy and Definitions for Terms Related to Driving

Automation Systems for On-Road Motor Vehicles (J3016 Ground Vehicle Standard). DOI :

http://dx.doi.org/https:

//doi.org/10.4271/J3016_201806

21. Stella Donker and Christian Janssen. 2018. What are questions and issues that we see about the mobile office? (2018). https://bit.ly/2HYUMSj Date accessed: 2019-06-06.

22. Consuelo Valdes, Diana Eastman, Casey Grote, Shantanu Thatte, Orit Shaer, Ali Mazalek, Brygg Ullmer, and Miriam K. Konkel. 2014. Exploring the Design Space of Gestural Interaction with Active Tokens Through User-defined Gestures. In Proceedings of the 32Nd Annual ACM Conference on Human Factors in Computing Systems (CHI '14). ACM, New York, NY, USA, 4107-4116. DOI :

http://dx.doi.org/10.1145/2556288.2557373

23. Hidde van der Meulen, Andrew L. Kun, and Orit Shaer. 2017. What Are We Missing?: Adding Eye-Tracking to the HoloLens to Improve Gaze Estimation Accuracy. In
Proceedings of the 2017 ACM International Conference on Interactive Surfaces and Spaces (ISS '17). ACM, New York, NY, USA, 396-400. DOI :

http://dx.doi.org/10.1145/3132272.3132278

24. Tamara von Sawitzky, Philipp Wintersberger, Andreas Riener, and Joseph L. Gabbard. 2019. Increasing Trust in Fully Automated Driving: Route Indication on an Augmented Reality Head-up Display. In Proceedings of the 8th ACM International Symposium on Pervasive Displays (PerDis '19). ACM, New York, NY, USA, Article 6,7 pages. DOI :

http://dx.doi.org/10.1145/3321335.3324947

25. Philipp Wintersberger, Andreas Riener, and Anna-Katharina Frison. 2016. Automated Driving System, Male, or Female Driver: Who'D You Prefer? Comparative Analysis of Passengers' Mental

Conditions, Emotional States \& Qualitative Feedback. In Proceedings of the 8th International Conference on Automotive User Interfaces and Interactive Vehicular Applications (Automotive'UI 16). ACM, New York, NY, USA, 51-58. DOI :

http://dx.doi.org/10.1145/3003715.3005410 